\title{
Belphégor
}

\section{La transition numérique de la bande dessinée franco-belge, une mutation impossible?}

\section{Raphaël Baroni, Gaëlle Kovaliv et Olivier Stucky}

\section{(2) OpenEdition}

1 Journals

\section{Édition électronique}

URL : https://journals.openedition.org/belphegor/3948

DOI : 10.4000/belphegor.3948

ISSN : 1499-7185

Éditeur

LPCM

\section{Référence électronique}

Raphaël Baroni, Gaëlle Kovaliv et Olivier Stucky, « La transition numérique de la bande dessinée franco-belge, une mutation impossible ? », Belphégor [En ligne], 19-1 | 2021, mis en ligne le 26 juin 2021, consulté le 08 juillet 2021. URL : http://journals.openedition.org/belphegor/3948 ; DOI : https:// doi.org/10.4000/belphegor.3948

\section{Ce document a été généré automatiquement le 8 juillet 2021.}

\section{c) (†) $९$}

Belphégor est mis à disposition selon les termes de la Licence Creative Commons Attribution - Pas d'Utilisation Commerciale - Pas de Modification 4.0 International. 


\title{
La transition numérique de la bande dessinée franco-belge, une mutation impossible?
}

\author{
Raphaël Baroni, Gaëlle Kovaliv et Olivier Stucky
}

1 Cet article ${ }^{1}$ propose d'interroger les effets de l'émergence des technologies numériques sur le champ de la bande dessinée de tradition franco-belge ${ }^{2}$. Alors que d'autres médias, tels que le cinéma, la télévision, la radio ou la musique enregistrée, ont été fortement reconfigurés suite à la démocratisation des outils numériques, qui ont transformé les modes de production, de diffusion et de consommation des contenus culturels, nous partirons du constat que la bande dessinée européenne semble opposer une certaine résistance face à cette mutation. La numérisation des œuvres du patrimoine francobelge et l'émergence de nouveaux genres nativement numériques demeurent ainsi des phénomènes relativement marginaux dans la production européenne.

2 Pour mieux comprendre ce phénomène, nous nous pencherons en particulier sur les processus historiques de reconfiguration des contenus entraînés par l'introduction des supports numériques, ainsi que sur la manière dont les nouvelles technologies transforment le marché, les pratiques et l'identité professionnelle des auteurs et des autrices. Ces deux aspects, l'un sémiotique et historique, l'autre social, permettront d'expliquer en partie pourquoi les œuvres numérisées ou nativement numériques n'ont pas supplanté une production mainstream qui demeure encore largement ancrée dans le dispositif du livre, qu'il s'agisse du format standard de l'album franco-belge ou de sa déclinaison plus récente labellisée "roman graphique ». Nous verrons en particulier que la numérisation de la bande dessinée, en transformant les interfaces de lecture, pose des problèmes spécifiques pour un mode d'expression fondé sur la mise en séquence d'un contenu narratif étalé dans l'espace graphique de son support. La variabilité du format et de la taille des supports de visionnage numérique entraîne ainsi des modifications sensibles du message, sans qu'il soit possible de remédier totalement à cette distorsion. Par ailleurs, au-delà de la difficulté technique entrainée par la numérisation des albums et des romans graphiques, un autre problème tient au statut 
culturel des supports reconfigurés, car nous verrons à quel point, historiquement parlant, le livre demeure une référence symbolique majeure dans le processus qui a conduit à l'autonomisation du champ de la bande dessinée dans le paysage culturel francophone de la seconde moitié du $\mathrm{Xx}^{\mathrm{e}}$ siècle.

Enfin, du point de vue des conditions matérielles et des représentations sociales des auteurs et des autrices, l'abandon des supports liés aux formats analogiques conduit à une alternative qui, d'une manière ou d'une autre, les éloigne du champ de la bande dessinée, que ce soit par nécessité économique ou par désir d'émancipation. D'un côté, du fait de l'absence de modèle économique viable, les œuvres les plus massivement diffusées numériquement - souvent via des blogs ou des réseaux sociaux - menacent l'autonomie économique des auteurs et des autrices; d'un autre côté, quand ces derniers ou ces dernières adoptent une posture plus expérimentale, ils ou elles refusent souvent le rattachement de leur production au champ de la bande dessinée pour éviter d'en brader les potentialités expressives. En effet, l'élargissement des moyens d'expression liés aux nouvelles technologies - effets sonores, animation, interactivité, etc. - induit le risque d'une dissolution de l'identité médiatique des "récits graphiques » (Chute, 2020), qui se confondent parfois avec des dessins animés, des jeux vidéo ou des dispositifs de réalité augmentée. Certains des auteurs ou des autrices aux pratiques les plus expérimentales assument alors cette émancipation du champ de la bande dessinée de manière à exploiter pleinement les potentialités des dispositifs numériques.

\section{Histoire des reconfigurations de la bande dessinée et des supports numériques}

4 Dans ses différents travaux sur l'histoire des supports de publication, Sylvain Lesage $(2018,2019)$ montre comment la bande dessinée franco-belge a construit son identité médiatique autour de la forme de l'album, reléguant d'autres supports dans les marges, malgré l'importance historique que ceux-ci ont pu représenter. Parmi ces supports marginalisés, il évoque notamment la presse périodique, qui a pourtant joué un rôle majeur dans la structuration de pratiques et de formes considérées comme déterminantes pour l'identité de la bande dessinée en tant que média autonome. En outre, ses recherches contribuent également à mettre au jour une variété de supports dont la marginalité apparente par rapport à l'album contraste avec leur importance historique et théorique. L'album de bande dessinée endosse ainsi un rôle fondamental dans la constitution médiatique de la bande dessinée franco-belge, notamment parce qu'il s'inscrit dans l'imaginaire symbolique du livre et qu'il a ainsi servi de véhicule à la légitimation et à l'institutionnalisation de ce médium dans l'espace culturel. Toutefois, cette importance symbolique doit être envisagée en rapport étroit avec un certain « nomadisme " éditorial de la bande dessinée, laquelle s'est toujours montrée prompte à explorer de nouveaux supports de publication, les problématiques contemporaines relatives à l'édition numérique s'inscrivant dans la continuité de cette histoire.

5 L'étude des reconfigurations découlant du transfert d'un support à un autre permet de souligner le rapport particulier qu'entretient la bande dessinée avec ses supports de publication. Ce phénomène est d'autant plus visible et central que la pratique consistant à publier un même récit sous différents formats traverse l'histoire éditoriale de la bande dessinée franco-belge. Du périodique à l'album, de l'album au CD-ROM, au 
livre de poche ou aux plateformes de lecture sur le Web en passant également par le contenu de blogs ou de plateformes en ligne transféré vers le livre, l'histoire de ces reconfigurations nous renseigne autant sur la diversité de telles pratiques éditoriales qu'elle permet de mettre en perspective les enjeux contemporains relatifs à la transition numérique de la bande dessinée.

6 La bande dessinée franco-belge se standardise comme forme médiatique dans le domaine de la presse durant la première moitié $\mathrm{du}_{\mathrm{xx}}^{\mathrm{e}}$ siècle et n'intègre solidement le secteur du livre qu'à partir des années 1970. Néanmoins, la position dominante de ce support, tant dans l'historiographie que dans l'imaginaire culturel de la bande dessinée, n'est pas accidentelle. Comme le montrent les recherches de Lesage, le passage à l'album est indissociable du processus de légitimation et d'institutionnalisation du média, et cela n'est pas sans lien avec le contexte particulier dans lequel s'opère actuellement (ou ne s'opère pas) la transition numérique. Autant parce que l'objet livre participe à la construction d'une "bande dessinée adulte ${ }^{3}$ ", que parce qu'il favorise l'émergence de la figure de l'auteur et qu'il permet d'installer le médium dans l'institution mémorielle d'une culture profondément biblio-centrée, le passage de la presse au livre marque ainsi l'entrée de la bande dessinée dans la mémoire culturelle de l'espace francophone. Cette situation contraste avec celle que l'on peut observer en Amérique du Nord ou en Asie, où l'émergence du roman graphique apparaît beaucoup plus tardive (vers la deuxième moitié des années 1980), et survient donc à une étape que l'on pourrait qualifier de post-légitimation, de sorte que la transition numérique du comic book ou du manga n'a pas été perçue comme une alternative entre le livre et sa lecture sur écran.

7 Les conséquences du passage du périodique à l'album ne sont cependant pas à comprendre du seul point de vue de l'histoire éditoriale. Les effets recherchés par les pratiques de narration en feuilleton, qui caractérise une bonne partie de la production destinée d'abord à la presse illustrée (Boillat et al., 2016), se trouvent parfois neutralisés par la forme continue et clôturée de l'album. Durant la période où commence à se systématiser une logique de double publication, le transfert des planches, strips ou demi-planches du magazine vers le format de l'album implique des opérations de reconfiguration pouvant aller du remontage de cases jusqu'à des interventions graphiques beaucoup plus poussées, transformant parfois assez radicalement le récit original. Au cours des années 1960, l'album passe progressivement, dans les stratégies éditoriales, du statut d'objet dont la seule fonction était de maximiser la rentabilité des publications tirées des périodiques, à celui d'aboutissement, plus ou moins prestigieux, pour les épisodes d'une série à succès. C'est ainsi que peut s'observer une évolution sensible des pratiques de création, lesquelles intègrent d'emblées les contraintes de l'album lors de la conception des planches publiées dans les hebdomadaires (Lesage, 2019, p. 85-93).

8 Ayant intégré le marché du livre et ses codes dès les années 1960, il pourrait paraître «naturel » que la bande dessinée franco-belge ait été confrontée au bouleversement qu'a entraîné le succès du livre de poche à la même époque. En raison des spécificités sémiotiques des récits graphiques, une telle évolution vers des formats réduits et plus économiques a cependant été entravée, cette divergence médiatique par rapport à l'évolution du champ littéraire apparaissant avec le recul comme une préfiguration des difficultés qu'entraînera plus tard la numérisation du patrimoine dans la bande dessinée franco-belge. Si l'édition de titres importés ou de volumes inédits en petit 
format a effectivement constitué un marché plus ou moins pérenne depuis les années 1950 (Lesage, 2019, p. 274-286), le transfert de l'album au format de poche est resté relativement marginal, les différentes tentatives dans ce sens ayant toutes échoué à plus ou moins brève échéance. Cela s'explique par le fait que la réédition de « classiques » en format poche, qui constitue le principal moteur de la « révolution » du livre de poche, a rencontré un obstacle dès lors que cette formule a été appliquée au domaine la bande dessinée 4 . En raison de son format réduit, le livre de poche constitue en effet un défi éditorial considérable pour l'adaptation de récits graphiques tirés de publications sous la forme d'albums. Les difficultés techniques inhérentes à une telle reconfiguration sont nombreuses, de la restriction des dimensions de la page aux problèmes de lecture que provoque une reliure de qualité médiocre.

9 Pour des questions de lisibilité des textes et des images, le transfert vers le livre de poche implique la recomposition des planches, une modification de la mise en page, voire un redécoupage des cases, ces retouches graphiques étant parfois si importantes que le résultat tend à questionner l'identité d'un médium comme la bande dessinée, dont la forme semble si fortement liée aux déterminations matérielles de son support. À l'inverse, la réduction paginale d'un roman ne transforme pas de manière fondamentale la composition du texte littéraire, qui se fonde sur des unités intégralement transposables: mots, phrases, paragraphes ou chapitres. Aussi, les enjeux formels, techniques et culturels liés à l'identité médiatique de la bande dessinée dans le cadre de la remédiatisation des albums pour les écrans résonnent d'autant plus clairement dès lors qu'ils sont mis en série avec d'autres cas de reconfiguration situés pourtant dans le paradigme commun de l'imprimé. L'échec du transfert de l'album vers le format de poche éclaire en quelque sorte les problèmes soulevés par leur numérisation et leur lecture sur des interfaces dont les formats doivent s'adapter à la diversité des écrans fixes et portables, certains d'entre eux n'excédant pas la surface moyenne d'une seule case de bande dessinée.

10 Comprendre la production et la consommation numériques de bande dessinée au $\mathrm{xxI}^{\mathrm{e}}$ siècle nécessite en premier lieu de différencier deux modes éditoriaux distincts. D'un côté, les différentes interfaces informatiques en usage (smartphones, ordinateurs personnels, tablettes...) sont envisagées comme un support alternatif pour lire une version numérisée d'un titre déjà paru en album. Dans ce cas de figure, les formats d'écrans ou la maniabilité de l'appareil employé s'avèrent plus ou moins (in)adaptés aux pratiques diverses de la lecture. De l'autre côté, l'hébergement sur le web d'espaces personnels (du site web développé et géré par un ou une internaute aux blogs) ou de plateformes d'échanges ( $\mathrm{du}$ forum aux «réseaux sociaux» en passant par les plateformes spécialisées) stimulent la production de contenus d'abord pensés pour la diffusion numérique. Comme le montre Julien Baudry (2018), ces deux paradigmes coexistent et parfois s'entrecroisent dès les années 1990, bien que l'auteur s'attarde plus particulièrement sur le second modèle, dans une volonté de constituer l'histoire de la bande dessinée numérique francophone. Si nous reviendrons, dans la deuxième partie de cette étude, sur le versant nativement numérique de la bande dessinée, il convient néanmoins de s'attarder sur le premier de ces deux paradigmes, qui permet de mettre en lumière les problématiques posées par le support informatique pour la publication de contenus d'abord pensés pour l'album imprimé, ou à l'inverse, la manière dont certains contenus d'abord publiés pour une lecture sur écran, intègrent malgré tout l'horizon du livre au moment de leur production. 
11 Dans les années 1990, l'arrivée de l'ordinateur personnel dans les foyers français constitue une révolution culturelle. Ce phénomène a suscité l'intérêt du milieu de l'édition de bandes dessinées pour ce qui est alors envisagé comme un nouveau support potentiel de publication. C'est notamment avec la popularisation du disque optique calibré pour le stockage de l'information (CD-ROM) que se met en place un nouveau paradigme éditorial dans lequel l'ordinateur personnel, le CD-ROM et l'imaginaire du multimédia se font les ingrédients d'un bouleversement possible des pratiques éditoriales. Moins de dix titres sont mis sur le marché entre 1995 et 1998. Malgré la marginalité d'une telle pratique, celle-ci est néanmoins révélatrice d'un bouleversement de paradigme au seuil de ce que l'on peut aujourd'hui qualifier de « tournant numérique " des industries culturelles, car elle constitue un laboratoire de solutions formelles pour publier des bandes dessinées sur des supports informatiques. En dépit de leur rareté, les albums reconfigurés sur CD-ROM mobilisent en effet de manière souvent expérimentale les mécanismes de l'interactivité, de l'hypermédialité ${ }^{\text {, }}$ l'ajout de sons et l'animation des contenus graphiques, que l'on retrouvera dans les bandes dessinées dites "nativement numériques" de la décennie suivante ${ }^{6}$. Mais ce sont sans doute les stratégies de désarticulation de la planche sous la forme du diaporama qui constituent autant la solution la plus fréquemment envisagée dans ce type de transferts que le mode de visualisation le plus pérenne dans le cadre des reconfigurations d'albums sur supports informatiques, notamment dans les cas de calibrage pour des écrans de tailles réduites tels que ceux des smartphones.

Dès le début des années 2000, l'accès à Internet devient de plus en plus courant dans le grand public. Le CD-ROM, dont l'heure de gloire n'aura finalement duré qu'une demi décennie, s'il conserve un rôle dominant pour la diffusion de logiciels payants et de jeux vidéo, se voit toutefois progressivement remplacé, d'abord par des supports physiques plus "performants» tels que les DVD-ROM, puis par les voies du téléchargement que facilitent les progrès dans le trafic des données, dont l'ADSL puis la fibre optique constituent des jalons importants. Si la publication de bandes dessinées nativement numériques connaît un engouement certain durant la première décennie des années 2000, on observe à l'inverse une baisse d'intérêt des éditeurs traditionnels pour la publication de leur catalogue imprimé dans des versions accessibles en ligne. L'ordinateur personnel, domestique ou nomade, ne semble pas représenter un support de publication viable pour un secteur qui se concentre alors sur la diversification des formes du livre. Dès les années 1990, on observe d'ailleurs l'émergence du roman graphique, un nouveau format de livre, moins standardisé que le traditionnel album franco-belge, qui achève le processus d'artification (Heinich, 2017) et d'élargissement du lectorat de la bande dessinée. Le prix Pulitzer du Maus d'Art Spiegelman, traduit et publié par Flammarion en France, le succès commercial et critique de Persepolis de Marjane Satrapi, ainsi que le développement des rayons consacrés à la bande dessinée dans les librairies généralistes, indiquent que l'avenir de la bande dessinée reste encore fortement lié à l'industrie du livre.

13 Ce n'est qu'à partir des années 2010, parallèlement à la popularisation des smartphones et des tablettes tactiles " connectées ", que se mettent en place plusieurs plateformes en ligne dont le but est de donner accès à des bandes dessinées numérisées, leur format pouvant éventuellement s'adapter à la diversification des écrans. Le lancement de la plateforme Ave!Comics par la société Aquafadas en 2008 constitue un point de départ important pour une telle entreprise éditoriale. Dans ce cas, l'initiative repose sur une 
société spécialisée dans le développement et la diffusion de logiciels, qui occupe ainsi une position extérieure vis-à-vis du monde de l'édition de bande dessinée. On trouve un modèle opposé deux ans plus tard lors de la mise en place de la plateforme Izneo, pilotée en amont par un consortium d'éditeurs associés de près ou de loin au groupe MédiaParticipations. Si Ave!Comics n'est aujourd'hui plus en activité, Izneo, associé à un puissant groupe d'édition-communication, constitue la plus importante plateforme de diffusion de bande dessinée franco-belge. Outre la diversité de son catalogue, l'un des principaux arguments de la plateforme réside dans les fonctions du logiciel « EazyComics » associé à une partie de son catalogue qui permet d'adapter les planches d'albums numérisés en fonction des contraintes inhérentes à la diversité des formats d'écrans sur lesquels peuvent être consultées les bandes dessinées. En plus de différents niveaux de zoom sur la simple ou la double planche, «EazyComics » offre la possibilité de (re)lire certains titres sous la forme d'un diaporama de cases, proposant ainsi une reconfiguration dynamisée de l'album imprimé en fonction de la diversité des supports numériques.

Parallèlement au développement de telles solutions, qui opèrent à une échelle industrielle, prend place un phénomène que l'on pourrait qualifier d'artisanal, celui d'auteurs et d'autrices publiant des travaux inédits sur leurs blogs personnels, avant d'être édités dans le circuit traditionnel du livre. Le phénomène des blogs BD opère un tournant autant pour la production de bande dessinée destinée à une publication pour l'écran que pour la constitution d'une nouvelle pratique éditoriale. En effet, de nombreux titres publiés en ligne trouvent un prolongement sous forme de livres imprimés par des éditeurs bien établis. Dans ces cas de figure, dont la publication par Albin Michel du Blog de Frantico en 2005 se fait le coup d'envoi, il est particulièrement frappant de constater qu'à l'instar de la modification des pratiques de création intervenue lorsque l'album est devenu l'horizon éditorial de la prépublication périodique, la publication des contenus sur les blogs $\mathrm{BD}$ tend progressivement à se standardiser autour de la planche verticale, alors même que le format standard de l'écran durant la seconde moitié des années 2000 était de forme horizontale. Cette standardisation de la production diffusée par les blogs indique ainsi que l'horizon éditorial des auteurs et des autrices qui investissent avec succès ce nouveau support numérique reste, in fine, le livre imprimé, qui continue d'imposer son format spécifique.

\section{Une définition sous tension de la bande dessinée numérique francophone}

15 Pour comprendre l'état actuel de la production de la bande dessinée numérique francophone, il est intéressant de se poser la question du volume du marché. Bien que relativement peu médiatisé, il serait pourtant «énorme », selon Luc Bourcier (2018), responsable de la plateforme en ligne Izneo. Plus de 150 millions d'albums seraient ainsi téléchargés chaque année et lus sur des interfaces numériques. Cela représenterait trois fois le marché de la bande dessinée papier, qui écoule annuellement environ 40 millions d'albums. Ces chiffres impressionnants laissent penser que la bande dessinée aurait achevé son tournant numérique et la «dématérialisation » de son patrimoine. En réalité, acteur d'un secteur qui cherche encore son public et sa rentabilité économique, Luc Bourcier a intérêt à articuler de tels chiffres, pour des raisons d'ordre symbolique et de légitimation d'un marché émergent, dont il est l'un 
des fers de lance. Il faut préciser en effet que l'écrasante majorité des albums qu'il inclut dans son calcul sont des scantrads ${ }^{7}$ illégaux, et rien ne garantit que ces œuvres gratuites massivement téléchargées sont effectivement lues. Le $1 \%$ restant, qui constitue le marché légal, concerne aussi des albums préalablement publiés en format papier. Ainsi, même lorsque les gens lisent en ligne, le livre demeure la source du contenu numérisé. D'autant que, si l'on inverse la lunette, un rapport de 2017 de l'organisme Hadopi ${ }^{8}$ nous apprend que seuls $4-5 \%$ des lecteurs et des lectrices de bandes dessinées physiques sont également des lecteurs et des lectrices numériques. Dans les pratiques et les intérêts du public, le papier fait donc de la résistance, même dans un monde où foisonne la numérisation illégale des produits culturels. Dans ce paradigme hégémonique, la part de la création destinée en première instance à une lecture et une diffusion sur écran - la bande dessinée nativement numérique - demeure largement marginale.

16 À première vue, la bande dessinée, à l'inverse de certains médias comme la musique, le cinéma ou la photographie, n'a donc pas opéré une transition aussi radicale d'un support analogique vers un mode de diffusion presque intégralement numérique et en réseau. Depuis trois décennies, le champ de la bande dessinée a plutôt vu émerger une nouvelle branche en son sein, plus ou moins autonome ou expérimentale, tout en demeurant périphérique par rapport au marché du livre, qui continue de structurer l'identité médiatique des pratiques culturelles. Les modes de diffusion numériques ne concurrencent donc pas les supports analogiques, mais ils enrichissent les modes d'expression de la bande dessinée en introduisant de nouveaux formats.

Pour Pascal Robert, l'adaptabilité de la bande dessinée à différents supports apparaît comme une constante dans l'histoire du médium, et les reconfigurations qui en découlent ne remettent pas fondamentalement en question son identité : «la bande dessinée a réussi à se transporter et à se transposer sur des supports variés et donc à s'adapter, tout en maintenant une forte exigence au maintien global de son dispositif sémiotique, c'est-à-dire sans se trahir » (Robert, 2014, p. 46). C'est d'ailleurs dans la continuité de ce constat que certains créateurs et créatrices de bandes dessinées numériques conçoivent leur travail, comme un langage qui ne change pas quel que soit le support. Emmanuel Espinasse se positionne ainsi : «J'ai une vision assez élargie de la bande dessinée. Pour moi, ça ne tient pas dans un format comme le livre, ou même, je pense que ça peut tout à fait se déployer sous des formes qui n'existent pas encore. Et pour moi le numérique fait partie de ces espaces dans lesquels la narration séquentielle - c'est comme ça que je vois la bande dessinée - peut s'insérer ${ }^{* 9}$.

D'autres lectures sont toutefois possibles. Parmi eux, Anthony Rageul - chercheur et auteur de bandes dessinées numériques sous le pseudonyme de Tony - pense que la transition numérique n'est pas la dernière évolution formelle du neuvième art, mais qu'elle a donné lieu à l'apparition d'un nouveau médium (Rageul, 2014, p. 18). Selon lui, " c'est tout le Système de la bande dessinée qui est remis en cause par le passage au support numérique", car les nouvelles potentialités sémiotiques de ces supports déplacent certains traits définitoires du médium. Il ajoute que la bande dessinée numérique «tend un miroir à la bande dessinée en remettant en jeu tous les codes qui la gouvernent » (Rageul, 2014, p. 84) $)^{10}$.

19 Ce débat sur l'identité de la bande dessinée nativement numérique reflète une division des pratiques. Il existe en effet, dans les représentations des auteurs et des autrices, une multiplicité de conceptions et d'usages de la bande dessinée, qui se distribuent sur 
un large spectre. D'un côté, il y a celles et ceux pour lesquels la bande dessinée qui explore de nouveaux formats et territoires doit s'inscrire dans la continuité des formats historiques. De l'autre, on trouve les partisans et les partisanes de la revendication d'une autonomie et d'un nouveau statut pour le médium.

Parmi les tenants de la continuité, on retrouve les auteurs et les autrices pour qui l'utilisation de supports numériques répond en premier lieu à la volonté de toucher la plus vaste audience possible. C'est ce qu'explique David Revoy lorsqu'il raconte comment il s'est mis au blog: «Je me suis dit "c'est simple, si je veux être heureux, il suffit que je fasse des BD et que je les expose au plus grand nombre". J'avais déjà un petit blog où je faisais des tutoriels par rapport aux logiciels que j'utilisais. Il y avait déjà 1000 visites par jour. Je me suis dit "je vais leur montrer à eux ce que je fais". Dès la première j'ai eu la chance, c'est devenu viral ॥*. Jibé a un discours similaire : "Quand sont arrivés les blogs, je trouvais ça fascinant, car on pouvait publier ce qu'on voulait, notamment de la bande dessinée. Ça ne coûtait pas très cher et c'était disponible pour tout le monde très facilement et gratuitement $»^{*}$. La transition vers le numérique est donc avant tout utilitaire et ne remet ainsi pas en cause les codes du neuvième art, mais contribue à les adapter à un nouveau contexte de diffusion. C'est ainsi que se conçoivent la plupart des blogs BD qui ont émergé au milieu de la décennie 2000. Il ne s'agit bien sûr pas d'un mimétisme complet avec le livre et quelques différences visuelles existent. La plus saillante de ces différences s'observe notamment dans le fait d'organiser les cases sur une longue planche qui dépasse le cadre visuel et dont la lisibilité repose sur un mouvement de défilement vertical - adoptant en cela l'un des codes de base de la lecture écranique - plutôt que de recourir à une succession de pages de taille fixe. Néanmoins, la transition vers l'album papier se réalise la plupart du temps sans heurts majeurs, car les choix de découpage et de structuration restent souvent assez proches de ce que l'on trouve traditionnellement dans les formats imprimés.

21 Cette volonté de mettre l'accessibilité du récit graphique au centre du dispositif numérique se retrouve aussi dans des cuvres qui se distinguent davantage de l'organisation traditionnelle des cases au sein d'un espace tabulaire, comme par exemple: Les Autres Gens de Thomas Cadène. Cette œuvre monumentale, qui fait se succéder des cases au sein d'un diaporama, constitue une rupture formelle et un jalon dans l'histoire de la bande dessinée, tant par son inventivité que par le nombre d'auteurs et d'autrices impliqués. Néanmoins, Thomas Cadène, son créateur, déclare que son but n'est pas de «toucher les lecteurs de BD, mais plutôt les gens qui passent du temps derrière leurs écrans, et qui liraient leur épisode en prenant leur café le matin par exemple » (cité dans Vellin et Aigron, 2014, p. 38).

22 À l'opposé de cette vision du numérique conçue avant tout comme un support de diffusion à large échelle, d'autres approches visent à « déployer la "bande dessinée en ligne" comme une forme de dépassement de la bande dessinée traditionnelle » (Candel, 2009 , p. 125). Pour plusieurs auteurs et autrices, le support numérique offre une surface ludique où repousser les codes de la bande dessinée et tester des innovations formelles. Comme l'affirme Balak, pour ces bandes dessinées plus «conceptuelles, les outils numériques semblent devenir une fin en soi », la narration passant au second plan (cité dans Robert 2016, p. 18). Le livre ne représente alors plus un horizon ou une référence de base et la plupart des œuvres de Vidu, Tony, exaheva ou Emmanuel Espinasse sont conçues de telle sorte qu'elles sont de facto impubliables sur support analogique, car 
incluant une importante interactivité, des séquences animées et parfois des pistes sonores. Vincent Pianina explique aussi : "C'est justement parce que j'aime beaucoup les livres que je n'ai pas voulu faire des "planches scannées". J'ai voulu qu'il y ait un intérêt à ce que ce soit sur écran. Essayer de faire quelque chose qui n'aurait pas été possible sur papier, en faire quelque chose de spécial »*. Ce positionnement a motivé sa décision de faire de Scröll une œuvre « en une seule case, mais vraiment immense »*, à lire à l'aide d'un mouvement de défilement horizontal.

D'autres dispositifs, plus facilement transposables vers l'imprimé, sont aussi considérés comme situés en dehors du champ de la bande dessinée. C'est le cas du Turbomedia, terme inventé par Balak pour décrire un récit graphique qui se déploie par une succession de diapositives que le lecteur active en utilisant un bouton ou par le toucher. Ce type de bande dessinée numérique peut comporter du texte, des phylactères et, très rarement, de brefs sons ou de l'animation. Pour certains, l'absence de la coprésence des cases au sein d'une unité tabulaire de rang supérieur marque une rupture avec le langage de la bande dessinée. Ainsi que l'explique Jérôme Jouvray, « le Turbomedia est un nom rigolo pour un truc où on clique, mais qui n'est déjà plus de la bande dessinée. La bande dessinée, s'il n'y a pas de cases juxtaposées, c'en n'est plus » (cité dans Falgas, 2014b, p. 47). Cette rupture formelle avec les schémas de la bande dessinée papier peut parfois se doubler d'un rejet plus idéologique. Ainsi que l'explique Philippe Paolucci, Stephen Vuillemin, qui scinde clairement sa pratique papier de sa pratique numérique, fait état « d'un rejet des institutions et d'une vision désabusée du marché de la bande dessinée papier ». Vuillemin lui a ainsi confié que "c'est le manque de visibilité de la scène amateur qui l'a poussé à revoir [son] ambition première : publier une bande dessinée papier n'a finalement plus grand sens dans un tel contexte. Mieux vaut se focaliser sur le numérique » (cité dans Paolucci, 2016, p. 214).

Il existe de nombreuses positions intermédiaires entre le pôle de ceux qui prônent la continuité avec les formats traditionnels de la bande dessinée et le pôle de ceux qui veulent rompre avec les références culturelles au livre. En guise d'exemple, citons le positionnement de Yannis La Macchia, qui, s'il conçoit la bande dessinée numérique comme un espace d'exploration et d'expérimentations, s'inscrit en même temps très fortement dans le sillage de la bande dessinée papier. En sa qualité de fondateur et d'éditeur de la Collection RVB, qui publie uniquement des contenus numériques, il tient à affirmer la différence entre les supports : "Si l'œuvre est vraiment prévue pour l'écran, il est préférable de considérer les qualités propres au support plutôt que de restituer l'illusion de ce qu'il n'est pas ». En revanche, il reconnaît " qu'il est important de travailler avec les libraires, les bibliothécaires, la presse et l'ensemble de ce qui constitue la chaîne du livre " (La Macchia, 2018, p. 184). Il ne s'affranchit ainsi pas complètement de la matérialité physique de la bande dessinée, puisque toutes les œuvres de la Collection RVB sont accessibles numériquement grâce à un petit livret destiné à trouver sa place sur les rayonnages des bibliothèques.

La bande dessinée nativement numérique est donc un objet aux formes multiples, issu $\mathrm{du}$ livre, certes, mais également influencé par d'autres domaines. Ses auteurs et autrices convoquent ainsi fréquemment des références à d'autres médias, et principalement au monde de l'audiovisuel (Falgas, 2014a, p. 98). Cette orientation est particulièrement manifeste dans le cas de Les Autres Gens, œuvre pourtant historiquement et formellement centrale dans l'histoire du médium. Julien Falgas relève que « le fait que les collaborateurs réunis autour de $L A G$ se reconnaissent les uns 
les autres comme auteurs de bande dessinée est le seul lien initial de ce projet avec le cadre de référence de la bande dessinée » (Falgas, 2014a, p. 144). La focale n'est plus dirigée vers la page, mais vers l'écran : «Le choix explicite du scénariste, contenu dans l'appellation même "bédénovela" (qui rappelle le terme espagnol telenovela), a été de se référer non au monde de l'image imprimée, mais à l'esthétique de l'audiovisuel et, plus spécifiquement, du feuilleton télévisé » (Baudry, 2018, p. 322). Cette filiation est en effet explicite dans la forme même du projet, puisqu'il s'agissait d' épisodes" d'un " feuilleton » (les termes sont de l'auteur) diffusés quotidiennement sur internet ${ }^{11}$, avec des rappels des péripéties précédentes pour celles et ceux qui attraperaient le récit en cours de route. Simon Kansara et Emilie Tarascou, l'auteur et l'autrice de MediaEntity, se réfèrent également aux séries américaines, et même au cinéma en parlant des influences qui leur ont permis d'imaginer et de concrétiser leur projet (Falgas, 2014a, p. 98). Leur positionnement est d'autant plus intéressant que leur œuvre, un récit Turbomedia en plusieurs épisodes, à lire sur internet ${ }^{12}$ ou sur une application dédiée, «fait appel à de nombreuses potentialités du numérique, mais la parution d'albums papier chez Delcourt, à partir de 2014, est entièrement intégrée au projet numérique " (Baudry, 2018, p. 202). « Pour le Secret des cailloux qui brillent, au début on avait vraiment deux influences, deux totems : Adventure Time et Game of Thrones. Ce qui nous intéressait c'était le côté feuilleton et grosse histoire ${ }^{*}$ raconte de son côté Jeanne Balas. La référence à l'univers des jeux vidéo est aussi souvent mobilisée. Jeanne Balas cite également les jeux utilisant le système de point and click parmi les influences principales de ses bandes dessinées numériques. Samuel Pott liste quant à lui le jeu en ligne Banja.com au nombre de ses modèles*.

La raison de ce vaste panel de références est à chercher du côté de l'identité professionnelle des acteurs et des actrices du champ. En effet, bien que certaines et certains soient issus du monde de la bande dessinée traditionnelle, plusieurs créateurs et créatrices viennent du monde de l'audiovisuel et en particulier de l'animation. C'est notamment le cas d'Émilie Tarascou, mais, également, ce qui est encore plus parlant du fait de leur influence dans le domaine, d'Yves Bigerel (Balak) et d'Alexandre Ulmann (Malec), les deux créateurs du Turbomedia. D'autres, comme Emmanuel Espinasse ou exaheva, ont importé leur savoir-faire et leur univers de référence du monde du jeu vidéo, pour lequel ils ont initialement été formés. Cet ancrage initial d'une partie de ses acteurs et de ses actrices dans le milieu du multimédia et des productions audiovisuelles influence ainsi directement la création et l'évolution du champ de la bande dessinée numérique.

Cette hybridation de l'objet - il faudrait dire "des objets" tant les formes sont diverses ${ }^{13}$ - explique en partie l'absence de format fixe pour la bande dessinée numérique aujourd'hui et la difficulté de faire émerger de nouveaux standards commercialement viables. À de multiples reprises, le triomphe d'un dispositif a été annoncé, sans qu'il finisse par s'imposer comme un nouveau standard de la production numérique. En 2012, Thomas Cadène témoignait ainsi de la domination du diaporama (plus précisément, de la lecture case à case), alors que le standard était encore le modèle du défilement (ou scrolling) moins de deux ans auparavant, lorsque les blogs occupaient l'avant-scène de la bande dessinée numérique (Falgas, 2014b, p. 152).

Aujourd'hui, ces deux modèles semblent parvenus à une sorte de coexistence en position dominante, reléguant le mimétisme de la planche à une pratique plus marginale. L'avatar moderne de la bande dessinée en diaporama se trouve actuellement 
sur les réseaux sociaux, principalement sur Instagram. La plateforme, initialement prévue pour le partage de photos, permet en effet de poster jusqu'à dix images par publication, qui se succèdent l'une après l'autre. Simple d'utilisation et extrêmement populaire, l'application attire depuis quelques années de plus en plus de créateurs et de créatrices, notamment grâce au succès de la série Été, suivie par plus de 60000 personnes lors de sa parution quotidienne au cours de l'été 2017 (Baudry, 2018, p. 232). Paradoxalement, pour beaucoup, ce pan de la création en diaporama est, sous de nombreux aspect, l'héritier des blogs BD qui avaient consacré le modèle par défilement.

L'hégémonie des blogs étant révolue, ce sont les webtoons qui ont pris le relai de la bande "défilée ». Originaire de Corée du Sud, ce type de bande dessinée se conçoit comme une bande que l'on "scrolle » verticalement. Ces webtoons sont spécifiquement calibrés pour la lecture sur téléphone ou tablette via des applications dédiées et leur logique narrative est celle de la série, divisée en de nombreux épisodes très brefs qui paraissent à un rythme soutenu et régulier. Ce format est actuellement plébiscité en France, d'une part parce que l'interface est simple à mettre en place et à s'approprier, mais également parce que le succès dans son pays d'origine a de quoi faire rêver des éditeurs et éditrices, ainsi que les auteurs et autrices qui se lancent sur le marché. On considère en effet qu'en Corée du Sud, une personne sur trois lit un webtoon au moins une fois par semaine (Surcouf, 2018). Les autrices et les auteurs sont d'autant plus enclins à se laisser tenter par ce modèle qu'il s'agit actuellement d'une des très rares façons d'être rémunéré en produisant de la bande dessinée numérique, pour autant que l'on obtienne un contrat chez Delitoon, Webtoon Factory, Tapas ou Naver.

Les bandes dessinées diffusées sur Instagram et sur les plateformes de webtoon tendent ainsi à installer une standardisation de la production, qui apparaît comme une condition préalable pour le rattachement de ces formats de récits graphiques au champ de la bande dessinée. Néanmoins, en dépit de leur succès croissant, elles peinent à stabiliser sur le long terme un format éditorial commercialement viable, et il existe encore une multitude d'œuvres - à l'instar de celles d'exaheva, Vidu, Tony, Emmanuel Espinasse ou de la Collection RVB - qui se situent hors de ces paradigmes du diaporama ou du défilement. Cette multiplicité des formats est une spécificité de la production européenne francophone. En effet, les autres aires culturelles au sein desquelles la bande dessinée numérique est en plein essor, plus particulièrement les États-Unis et la Corée du Sud, ont tranché pour une forme stabilisée, respectivement le webcomic et le webtoon (Baudry et Paolucci, 2016, p. 59).

\section{Un média qui se distingue face à la transition numérique}

31 Dans cet article, nous avons pris le parti d'examiner la transition numérique de la bande dessinée du point de vue de son histoire médiatique spécifique et des enjeux qui se posent actuellement aux actrices et acteurs rattachés à ce champ. En cela, on pourrait nous reprocher d'exagérer les spécificités de cette transition et d'ignorer la manière dont la bande dessinée s'inscrit dans un système médiatique plus large. En effet, lorsqu'est envisagée la manière dont les technologies numériques ont transformé notre paysage culturel, de nombreux discours insistent sur la nécessité d'embrasser le phénomène sur une échelle qui transcende les frontières médiatiques traditionnelles. Pour Lev Manovich (2017), l'artiste contemporain étant libre de choisir et de combiner 
différents supports, le découpage du domaine de l'art en fonction de différents médiums (peinture, sculpture, photographie, etc.) ne reflèterait plus le fonctionnement réel de la culture. Dans une approche plus sociologique, Henry Jenkins a décrit quant à lui un mouvement de convergence médiatique observable au sein des industries culturelles, qui inviterait davantage à réfléchir à l'articulation entre de "multiples canaux de diffusion dans le but de créer une expérience de divertissement unifiée et coordonnée » (2020a, p. 177) plutôt qu'à envisager l'effet des technologies numériques sur chacun de ces canaux. Ainsi que l'affirme Jenkins, l'évolution culturelle qui a accompagnée l'entrée dans la culture en réseaux a conduit à «la mise en couche, la diversification et l'inter-connectivité des médias » (2020b, p. 183). Enfin, de Marshall McLuhan (1968) à Jay David Bolter et Richard Grusin (2000), l'évolution médiatique et la transition numérique ont été envisagées sous l'angle de l'inclusion des anciens médias dans les nouveaux, l'informatique interconnectée apparaissant comme l'aboutissement de la fusion des médias graphiques, textuels et audiovisuels au sein d'une même interface.

Entre l'approche de Manovich, qui souligne l'hybridation des pratiques culturelles, celle de Jenkins, qui insiste sur l'interconnectivité médiatique, et enfin celle de Bolter et Grusin, qui met l'accent sur les processus de remédiatisation ${ }^{14}$, il n'y a pas contradiction, mais la place occupée par un médium tel que la bande dessinée apparaîtra sensiblement différente. Le premier inviterait à prendre en considération la circulation du langage de la bande dessinée au sein d'œuvres contemporaines de plus en plus inclassables. Le second insisterait plutôt sur l'articulation de cette forme avec d'autres médias visant à produire une forme de divertissement unifiée, souvent dans le but de capter l'attention d'un segment du public: on sait l'importance de la bande dessinée dans la culture fanique, ainsi qu'en témoignent l'ancrage historique des ComicCon et l'origine des franchises Marvel et DC Comics. Enfin, les derniers mettraient plutôt en évidence l'accessibilité de contenus assimilables au langage de la bande dessinée au sein d'interfaces numériques incluant d'autres formes médiatiques, le processus étant censé enrichir les potentialités expressives de la forme remédiatisée en exploitant les possibilités techniques du nouveau support.

Quel que soit l'intérêt de ces diverses perspectives, il nous semble que nous avons montré qu'elles ne sont pas dépourvues d'angles morts lorsqu'il s'agit d'envisager, du point de vue d'un média pris comme point de référence, la manière dont les technologies numériques ont transformé l'industrie culturelle, les pratiques et les représentations des acteurs et des actrices. Philippe Marion et André Gaudreault ont souligné le fait que les médias sont en perpétuelle évolution, de sorte que l'émergence de nouvelles technologies, de nouveaux dispositifs et de nouvelles pratiques culturelles aboutit parfois à l'avènement d'une nouvelle identité médiatique :

Par des glissements ou des modifications de pratiques, par des changements socioéconomiques, etc., le média en vient donc à révéler certaines de ses spécificités expressives (communicationnelles, esthétiques, génériques, etc.). Cette ouverture à l'autonomie est en interrelation avec l'évolution du média et de son potentiel propre. Et lorsque affirmation identitaire et autonomie coïncident avec une reconnaissance institutionnelle et une amélioration décisive des ressources économiques de production se manifeste alors la deuxième naissance - l'avènement. (Marion \& Gaudreault, 2000, p. 35)

Si cela fait plusieurs décennies que l'émergence d'une bande dessinée numérique (ou numérisée) fait l'objet de discours et de tentatives artistiques et commerciales dans les 
pays francophones, force est de constater que l'avènement d'une nouvelle identité médiatique n'a pas eu lieu, ou du moins pas entièrement. Si l'on compare la situation avec le champ des productions audiovisuelles ou musicales, on constate que pour ces dernières, les technologies numériques n'ont pas seulement modifié les modes de production (avec l'apparition de nouveaux instruments électroniques, d'effets spéciaux engendrés informatiquement et de nouvelles techniques de captation) et de diffusion (avec le visionnage ou l'écoute sur une multitude de plateformes interconnectées), mais elles ont proprement révolutionné l'industrie culturelle elle-même, en remettant en cause les liens entre leur modèle économique et des supports qui ont été longtemps considérés comme définitoires de médias: la salle obscure pour le cinéma et les supports matériels (disques vinyle ou $\mathrm{CD}$ ) pour l'industrie « du disque ».

Pourtant, paradoxalement, du point de vue des consommateurs, ces changements apparaissent beaucoup moins visibles et radicaux que ceux observables dans le secteur de la bande dessinée numérique. Ces changements ont été moins visibles, parce qu'entre les œuvres musicales ou audiovisuelles reconfigurées par les technologies numériques et les œuvres originales, la transition s'est faite par intégration successive de techniques qui ne remettaient pas en cause fondamentalement l'identité du médium. Par ailleurs, avec l'augmentation des capacités de stockage, des flux de données et des vitesses de traitement de l'information, la remédiatisation des œuvres filmiques et musicales n'a posé aucun problème technique insurmontable. On peut affirmer aujourd'hui que, même si l'expérience esthétique diffère sensiblement, Citizen Kane d'Orson Welles ou The Wall de Pink Floyd restent fondamentalement les mêmes œuvres, quel que soit le mode de visionnage ou d'écoute. Cette transférabilité des œuvres explique que la transition numérique ait pu s'opérer sans obstacles insurmontable pour un transfert intégral du médium dans l'ère numérique.

Le faible succès des liseuses électroniques soulève le cas particulier de la littérature, dont l'attachement au support analogique du livre semble beaucoup plus fort que dans le domaine cinématographique et musical ${ }^{15}$. Dans le cas des textes littéraires, il semblerait donc que la lecture sur papier soit encore considérée comme une expérience insurpassable, les enrichissements promis par les liseuses électroniques en termes de portabilité de la bibliothèque, de navigation, d'annotation ou de documentation des œuvres ne semblant pas en mesure de transformer radicalement le marché. Umberto Eco a ainsi pu affirmer que le livre était à ses yeux une invention "parfaite", aussi indépassable que la roue et donc pratiquement irrémédiable au sens de Bolter et Grusin $^{16}$. Si une telle affirmation paraît discutable, elle n'en demeure pas moins le symptôme d'une résistance de certains secteurs de la culture à la transition numérique - du moins en ce qui concerne les supports de diffusion, on ne parle pas ici de la rédaction et de l'édition - que l'on ne peut mettre uniquement sur le compte d'un conservatisme esthétique. L'importance de la dimension symbolique du livre pour un champ culturel bâti autour de ce support se constate aussi par le fait que, comparativement parlant, les genres de la presse écrite (journaux d'information ou scientifiques) ont opéré un virage beaucoup plus marqué du côté des supports numériques.

37 Le cas de la bande dessinée apparaît encore plus emblématique de l'existence de spécificités locales qui s'opposent à un transfert complet de certains médias vers les interfaces numériques. Les changements de formats imposés par la variabilité des écrans apparaissent ainsi incompatibles avec le maintien de l'architecture graphique 
des planches, sur laquelle repose une part non négligeable de l'esthétique des récits graphiques, notamment dans la culture franco-belge centrée autour de l'album. Cette spécificité formelle a conduit à une différenciation beaucoup plus marquée que dans d'autres médias entre les contenus reconfigurés par un processus de numérisation des œuvres du patrimoine et les contenus que nous avons qualifiés de nativement numériques.

Pour les premiers, nous avons rappelé que l'album constitue encore, symboliquement et économiquement, un format de référence pour la bande dessinée franco-belge. Dans ce cas, la transition vers différents formats écraniques - d'abord l'écran de l'ordinateur, via les CD-ROM, puis celui des tablettes et des smartphones, via les plateformes de lecture en ligne - a conduit à modifier en profondeur les œuvres remédiatisées. Quel que soit le succès commercial de ces dispositifs, qui visent à diffuser le patrimoine numérisé des éditeurs franco-belges, ils se heurtent aux mêmes écueils que ceux rencontrés lors de tentatives antérieures visant à établir des formats de poche: l'impossibilité de restituer sans distorsion l'articulation d'une œuvre produite pour un format différent et la difficulté d'offrir une remédiatisation aussi satisfaisante, sur un plan esthétique, que l'expérience offerte par le support livresque autour duquel s'est construite la légitimité médiatique de la bande dessinée.

De fait, si la bande dessinée numérique finit un jour par rencontrer un succès suffisamment probant pour faire évoluer l'identité du médium, ce sera probablement par le biais d'une production nativement numérique instaurant un nouveau standard, dont la rentabilité pourra être comparée avec celle des formats de l'album et du roman graphique et qui pourra également prétendre à un certain prestige culturel. Là encore, l'analyse des œuvres contemporaines et des discours des acteurs et des actrices laisse entrevoir un paysage contrasté. D'un côté, on observe le développement d'œuvres expérimentales qui se rattachent clairement au champ de la production restreinte, mais qui, dans la lignée des propos de Manovich sur le post-média, ne revendiquent plus nécessairement leur rattachement au champ de la bande dessinée. De l'autre, c'est l'émergence d'une production commerciale sous forme de webtoons qui paraît la plus apte à induire une "reconnaissance institutionnelle » fondée sur une "amélioration décisive des ressources économiques de production» (Marion \& Gaudreault, 2000, p. 35). Mais là encore, l'avènement de cette nouvelle identité médiatique se heurte au caractère encore émergent de ces formats et à un déficit de légitimité culturelle des œuvres, qui contraste avec une production européenne qui s'est organisée depuis un demi-siècle autour du format du livre, lequel bénéficie encore d'un grand prestige dans le champ voisin de la littérature.

\section{BIBLIOGRAPHIE}

BAETENS J., (2020), « La remédiatisation : formes, contextes, enjeux », in R. BARONI, C. GUNTI (Dir.), Introduction à l'étude des cultures numériques. La transition numérique des médias, Malakoff, Armand Colin, p. 237-250. 
BAUDRY J., PAOLUCCI P., (2016), « La bande dessinée numérique vue d'ailleurs », in P. ROBERT (Dir.), Bande dessinée et numérique, Paris, CNRS Editions, p. 59-78.

BAUDRY J., (2018), Cases·Pixels. Une histoire de la BD numérique en France, Tours, Presses universitaires François-Rabelais.

BOLTANSKI L., (1975), La constitution du champ de la bande dessinée, Actes de la recherche en sciences sociales, $\mathrm{n}^{\circ} 1$, p. 37-59.

BOLTER J. D., GRUSIN R., (2000), Remediation, Cambridge (MA), MIT Press.

BOUDISSA M., (2016), « Typologie des bandes dessinées numériques », in P. ROBERT (Dir.), Bande dessinée et numérique, Paris, CNRS Éditions, p. 79-100.

BOURCIER L., (2018), Table ronde 2 : vers la réinvention des nouveaux modes de diffusion ?, La BD numérique, hors des cases?, Les 7èmes Rencontres de l'Édition numérique, Bruxelles, 17 mai.

BOILLAT A., BOREL M., CESTERLÉ R., REVAZ, F., (2016), Case, strip, action! Les feuilletons en bande dessinée dans les magazines pour la jeunesse (1946-1959), Lausanne, InFolio.

CANDEL E., (2009), La bande dessinée en ligne, entre idéaux de rupture et de continuité, Hermès La Revue, $\mathrm{n}^{\circ}$ 54, p. 125-126. [En ligne] Disponible à l'adresse : https://www.cairn.info/revue-hermesla-revue-2009-2-page-125.htm (consulté le 13/01/2021).

CARRIÈRE J.-C., ECO U., DE TONNAC J.-P., (2009), N'espérez pas vous débarrasser des livres, Paris, Grasset.

CHUTE H., ([2008] 2020), La bande dessinée est-elle de la littérature ? Lire les récits graphiques, Transpositio. [En ligne] Disponible à l'adresse : http://www.transpositio.org/articles/view/labande-dessinee-est-elle-de-la-litterature-lire-les-recits-graphiques (consulté le 13/01/2021).

FALGAS J., (2014a), « Raconter à l'ère numérique. Auteurs et lecteurs héritiers de la bande dessinée face aux nouveaux dispositifs de publication », Thèse de doctorat : Sciences de l'Information et de la Communication, Université de Lorraine.

FALGAS J., (2014b), « Raconter à l'ère numérique. Auteurs et lecteurs héritiers de la bande dessinée face aux nouveaux dispositifs de publication. Tome 2 - Annexes ", Thèse de doctorat : Sciences de l'Information et de la Communication, Université de Lorraine.

GAUDREAULT A., MARION P., (2000), Un média naît toujours deux fois..., Sociétés et représentations, vol. $9, \mathrm{n}^{\circ}$ 2, p. 21-36. [En ligne] Disponible à l'adresse : https://www.cairn.info/revue-societes-etrepresentations-2000-2-page-21.htm (consulté le 13/01/2021).

HEINICH N., (2017), L’artification de la bande dessinée, Le Débat, nº 195, p. 5-9.

JENKIN H., (2020a), « Dix thèses sur la narration transmédiatique », in R. BARONI, C. GUNTI (Dir.), Introduction à l'étude des cultures numériques. La transition numérique des médias, Malakoff, Armand Colin, p. 177-181.

JENKIN H., (2020b), « Quelques réflexions supplémentaires sur le transmédia », in R. BARONI, C. GUNTI (Dir.), Introduction à l'étude des cultures numériques. La transition numérique des médias, Malakoff, Armand Colin, p. 183-192.

LA MACCHIA Y., (2018), Pour une bande dessinée numérique de création, Revue Bédéphile, $\mathrm{n}^{\circ}$ 4, $\mathrm{p}$. 178-185.

LEMOINE C., (2012), Poche, Neuvième art 2.0. [En ligne] Disponible à l'adresse : http:// neuviemeart.citebd.org/spip.php?article510 (consulté le 12/01/2021). 
LESAGE S., (2018), Publier la bande dessinée : les éditeurs franco-belges et l'album, 1950-1990, Villeurbanne, Presses de l'ENSSIB.

LESAGE S., (2019), L'Effet livre : métamorphoses de la bande dessinée, Tours, Presses universitaires François-Rabelais.

MANOVICH L., ([2017] 2001) Une esthétique post-média, Appareil, $\mathrm{n}^{\circ} 18$. [En ligne] Disponible à l'adresse : http://journals.openedition.org/appareil/2394 (consulté le 13/01/2021).

MCLUHAN M., ([1968] 1964), Pour comprendre les médias, Paris, Seuil.

PAOLUCCI P., (2016) «La bande dessinée numérique : entre rupture et continuité Pour une approche socio-sémiotique de la transition numérique du neuvième art : l'exemple de la revue en ligne Professeur Cyclope. Tome 2 - Annexes ", Thèse de doctorat : Sciences de l'Information et de la Communication, Université Lumière Lyon 2.

RAGEUL A., (2014), « La bande dessinée saisie par le numérique : formes et enjeux du récit reconfiguré par l'interactivité », Thèse de doctorat : Art et histoire de l'art, Université Rennes 2.

ROBERT P., (2014), La bande dessinée, une subversion sémiotique des supports de l'intermédialité ?, Communication \& langages, vol. 4, n¹82, p. 45-59.

ROBERT P., (2016), (Dir.), Bande dessinée et numérique, Paris, CNRS Éditions.

ROBERT P., (2016), « Le point de vue des auteurs », in P. ROBERT (Dir.), Bande dessinée et numérique, Paris, CNRS Éditions, p. 173-196.

SURCOUF O., (2018), Le webtoon, la bande-dessinée numérique coréenne qui cartonne, Le Figaro, 9 février.

VELLIN C., AIGRON G., (2014), « Usages et pratiques de la bd numérique », Mémoire de Master 2 : Marketing, Kedge Business School, Marseille.

\section{NOTES}

1. Cette réflexion a été développée dans le cadre du projet Sinergia «Reconfiguring Comics in our Digital Era", financé par le Fonds national suisse de la recherche scientifique (FNS: CRSII5_180359).

2. Sur l'émergence en France de ce « champ » à partir des années 1960, nous renvoyons à l'article pionnier de Boltanski (1975).

3. La notion est à comprendre autant au niveau du public visé qu'à celui des thématiques abordées. La diversité des aspects découlant de cette formule est amplement développée chez Lesage (2019, p. 253-271).

4. Pour un panorama historique détaillé de ces expériences éditoriales voir Lemoine 2012 ; Lesage 2019, p. 305-339.

5. La notion d'hypermédialité est ici à comprendre comme variante de la logique hypertextuelle. Bien que le terme n'ait pas connu un destin aussi marquant que celui d'hypertexte, il sert toutefois à distinguer plus précisément ce qui relève d'un système de liens fondés sur des données textuelles de ce qui permet de mettre en communication différentes sources médiatiques (texte, son, image, vidéo, etc.).

6. À titre d'exemple, on mentionnera Le Piège diabolique (Index+ et France Télécom Multimédia, 1997) qui inclut deux versions reconfigurées (l'une « interactive » et l'autre "commentée ») de l'album d'Edgar P. Jacobs. Ces deux adaptations sur CD-ROM mobilisent diversement les différentes stratégies de reconfiguration sur support numérique. 
7. Fruits du travail bénévole de fans, les scantrads sont la numérisation - qui s'accompagne souvent d'une traduction réalisée par leurs soins, dans le cadre des albums inédits en France - et la diffusion à large échelle de leurs œuvres favorites sur internet.

8. Hadopi, «La diffusion dématérialisée de BD et de mangas en France : rapport définitif », 22 décembre 2017, [en ligne], url: https://hadopi.fr/sites/default/download/ HADOPI_Rapport_BD_numerique.pdf.

9. Toutes les citations suivies d'un astérisque proviennent d'un corpus d'entretiens menés par nos soins auprès de plus de trente créateurs et créatrices de bandes dessinées numériques entre septembre 2019 et janvier 2020.

10. Ce type de renégociation, nous l'avons vu, n'est cependant pas un apanage de la transition numérique, l'album ayant également remodelé considérablement l'identité du médium.

11. http://www.lesautresgens.com

12. http://www.mediaentity.net

13. Pour une description des principaux modèles formels, nous renvoyons à la typologie établie par Magali Boudissa (2016, p. 79-100). Il existe, en gros, trois grands modèles, qui ont eux-mêmes plusieurs déclinaisons : le strip (ou webcomic), le diaporama (ou Turbomedia), le défilement (ou scrollcomics). Nous en ajoutons un quatrième: le "mimétisme planche", lorsqu'il s'agit de planches de bande dessinée « classique » dont la seule particularité numérique est la diffusion en première instance sur internet.

14. Sur le choix de cette traduction, dont la connotation est plus neutre que le terme « remédiation », voir Baetens (2020).

15. Plus qu'une réelle résistance, le retour relatif des disques vinyles trahit plutôt une reconfiguration locale du champ, la fétichisation de l'objet analogique ne concernant que le champ de la production restreinte. Quant à la survivance des salles de cinéma, elle s'est faite au prix d'une évolution technologique qui consiste à les placer à l'avant-garde technologique du spectacle audio-visuel en 4DX, ce qui renvoie à la conception d'un cinéma-attraction.

16. Voir les propos tenus par Eco dans l'ouvrage d'entretiens menés par Philippe de Tonnac (Carrière, Eco \& Tonnac 2009).

\section{RÉSUMÉS}

Cet article propose d'interroger la transition numérique de la bande dessinée franco-belge. Alors que d'autres médias ont été fortement reconfigurés par le numérique, nous montrerons que la bande dessinée semble opposer une résistance. L'étude historique des supports de publication permettra, dans un premier temps, d'éclairer les processus de reconfiguration liés à la multiplicité des supports de publication analogiques et numériques. Cette partie historique soulignera l'importance symbolique du rattachement de la bande dessinée au support du livre et les obstacles qui s'opposent à son transfert vers les écrans. Une seconde partie, fondée sur une analyse des représentations des acteurs, montrera comment les technologies numériques ont transformé le marché, les pratiques et l'identité professionnelle des auteurs et des autrices. Nous verrons à quel point le livre demeure une source de revenus difficilement remplaçable et une référence symbolique majeure pour un média dont le patrimoine ne se laisse pas facilement transférer sur des interfaces numériques. En l'absence de véritables alternatives économiques et du fait d'une émancipation médiatique des auteurs contemporains les plus créatifs, les 
déclinaisons numériques de la bande dessinée ne parviennent, ainsi, pas à transformer en profondeur le champ de production.

\section{INDEX}

Mots-clés : bande dessinée, livre, blog, CD-ROM, webtoon, turbomedia

\section{AUTEURS}

\section{RAPHAËL BARONI}

Raphaël Baroni est professeur associé à I'Université de Lausanne. II a publié La tension

narrative (Seuil, 2007), L'oeuvre du temps (Seuil, 2009) et Les rouages de I'intrigue (Slatkine, 2017). II a aussi codirigé une douzaine d'ouvrages collectifs ou de numéros de revue, parmi lesquels Introduction à l'étude des cultures numériques (Armand Colin, 2020) et Les avatars du chapitre en bande dessinée (Cahiers de narratologie, $n^{\circ} 34,2018$ ).

Il est cofondateur du Réseau des narratologues francophones (RéNaF: https://wp.unil.ch/narratologie/), du Groupe d'étude sur la bande dessinée (GrEBD: https://wp.unil.ch/grebd) et du Pôle de narratologie transmédiale (NaTrans : https://wp.unil.ch/narratologie/natrans/). II préside actuellement I'Association internationale des chercheurs en littératures populaires et culture médiatique (LPCM: https://lpcm.hypotheses.org/).

raphael.baroni@unil.ch

\section{GAËLLE KOVALIV}

Gaëlle Kovaliv est diplômée en français moderne et en sciences du langage à l'Université de Lausanne et mène une thèse de doctorat sur la bande dessinée numérique franco-belge dans le cadre du projet interdisciplinaire Reconfiguring comics in our digital era financé par le Fonds National Suisse de la recherche scientifique (https://wp.unil.ch/ grebd/ reconfiguring-comics/).

Elle est membre du Groupe d'étude sur la bande dessinée (GrEBD:https:// wp.unil.ch/grebd) et fait partie du comité éditorial de la revue Comicalités. Sa dernière publication est un lexique bilingue français-anglais des unités significatives de la bande dessinée.

gaelle.kovaliv@unil.ch

\section{OLIVIER STUCKY}

Olivier Stucky mène une thèse de doctorat sur les reconfigurations narratives dans la bande dessinée franco-belge à l'aune des variations de supports dans le cadre du projet interdisciplinaire Reconfiguring comics in our digital era financé par le Fonds National Suisse de la recherche scientifique (https://wp.unil.ch/ grebd/reconfiguring-comics/). Ses axes de recherche se situent à la convergence de la narratologie, de la théorie et de l'histoire de la bande dessinée. 
Il est membre du Groupe d'étude sur la bande dessinée (GrEBD: https:// wp.unil.ch/grebd), Réseau des narratologues francophones (RéNaF: https:// wp.unil.ch/narratologie/) et du Pôle de narratologie transmédiale (NaTrans : https://wp.unil.ch/narratologie/natrans/).

olivier.stucky@unil.ch 